\title{
DTX3L is upregulated in glioma and is associated with glioma progression
}

\author{
PENG XU ${ }^{1,2^{*}}$, XUELEI TAO ${ }^{2,3^{*}}$, CHENGJIN ZHAO $^{2,3}$, QINGFENG HUANG ${ }^{4}, \mathrm{HAO} \mathrm{CHANG}^{2,5}$, \\ NA BAN ${ }^{1}$, YUANQI BEI ${ }^{2}$, XIAOJIE XIA ${ }^{2}$, CHAOYAN SHEN $^{2}, \mathrm{KUN}_{\mathrm{WANG}}{ }^{1}, \mathrm{LI} \mathrm{XU}^{2}$, \\ PEIZHANG WU ${ }^{2}$, JIANBING REN ${ }^{3}$ and DONGLIN WANG ${ }^{1}$ \\ ${ }^{1}$ Department of Pathology, Nantong University; ${ }^{2}$ Jiangsu Province Key Laboratory for Inflammation and Molecular Drug Targets, \\ Nantong University; ${ }^{3}$ Department of Neurosurgery, The Second People's Hospital of Nantong; ${ }^{4}$ Department of Neurosurgery, \\ The Affiliated Hospital of Nantong University, Nantong, Jiangsu 226001; ${ }^{5}$ Department of Neurosurgery, \\ Wuxi Second Hospital Affiliated to Nanjing Medical University, \\ Wuxi, Jiangsu 214002, P.R. China
}

Received August 8, 2016; Accepted May 19, 2017

DOI: $10.3892 /$ ijmm.2017.3023

\begin{abstract}
Gliomas are the most common primary brain tumors of the central nervous system (CNS). Due to the poor prognosis of glioma patients, it is urgent to develop more effective therapies. Deltex-3-like (DTX3L), also known as B-lymphoma and BAL-associated protein (BBAP), has been reported to play an important role in the progression of many tumors. This study aimed to investigate the clinical significance and biological function of DTX3L in human glioma. Clinically, the protein expression level of DTX3L is increased in glioma tissues compared with that observed in normal brain tissues. Immunohistochemical analysis demonstrated that DTX3L was highly expressed in the glioma tissues and its level was correlated with the grade of malignancy. Multivariate analysis revealed the association between high expression of DTX3L and the poor prognosis of glioma patients. In addition, knockdown of DTX3L by siRNA transfection increased glioma cell apoptosis. Moreover, suppression of DTX3L expression was shown to significantly inhibit the migration and invasion of glioma cells. These data indicate that DTX3L plays an important role in the patho-
\end{abstract}

Correspondence to: Dr Jianbing Ren, Department of Neurosurgery, The Second People's Hospital of Nantong, 43 Xinglong Road, Nantong, Jiangsu 226001, P.R. China

E-mail: jianbingren288@sina.com

Dr Donglin Wang, Department of Pathology, Nantong University, 19 Qixiu Road, Nantong, Jiangsu 226001, P.R. China

E-mail: donglin@ntu.edu.cn

*Contributed equally

Key words: Deltex-3-like, glioma, migration, apoptosis genic process of glioma, suggesting that DTX3L could be a potential prognostic biomarker for glioma.

\section{Introduction}

Astrocytic brain tumors, the most common type of primary malignant brain tumors, are typically characterized by diffuse infiltrative growth and a low survival rate (1-3). Despite improvements in GBM therapy consisting of surgical resection and chemoradiotherapy with temozolomide (TMZ), only $10 \%$ of these patients survive 5 years after diagnosis and the majority of patients have a median survival of only 12-14 months (4-6). Thus, in order to identify potential diagnostic and therapeutic targets, it is urgent to understand the underlying molecular events which lead to the progression of glioma.

Deltex-3-like (DTX3L), also known as B-lymphoma and BAL-associated protein (BBAP), belongs to the Deltex (DTX) family (7,8). As an E3 ligase, DTX3L has been reported to regulate ESCRT-0 ubiquitination by inhibiting the activity of AIP4 (9). In addition, DTX3L exerts an effect on the DNA damage response pathway by modulating monoubiquitination of histone H4 (10). DTX3L was also identified as a binding partner of $\mathrm{B}$ aggressive lymphoma 1 (BAL1), a risk-related gene and protein in diffuse large B cell lymphoma (DLBCL) (11). Furthermore, it has been reported that DTX3L is overexpressed in melanoma and regulates melanoma metastasis via the FAK/PI3K/AKT pathway (12). Recently, DTX3L was also shown to play a role in prostate cancer cells via inhibiting IRF1 expression and its depletion was found to be related with tumor cell proliferation (13). All of these studies demonstrate that DTX3L is a novel biomarker with which to predict the progression of tumors. Nevertheless, to the best of our knowledge, the function of DTX3L has not yet been reported in gliomas.

In the present study, we evaluated the expression of DTX3L in human glioma tissues and explored its role in the migration and apoptosis of glioma cells. These findings may be useful to identify a new therapeutic target for glioma. 


\section{Materials and methods}

Primary human glioma specimens. All human glioma specimens were collected from 96 patients between 2007 and 2012 at a single institution, the Department of Pathology, Affiliated Hospital of Nantong University. All tumors were from patients with newly diagnosed gliomas who had received no therapy before sample collection. Normal brain tissues were obtained from patients undergoing surgery for epilepsy. All tissue samples were obtained using protocols approved by the Ethics Committee of the Affiliated Hospital of Nantong University. Tissues were frozen immediately after surgery and stored at $-80^{\circ} \mathrm{C}$ until use. For immunoblot analysis, all the tissues were frozen in liquid nitrogen immediately after surgery. The specimens for histological examination were fixed in formalin and embedded in paraffin for sectioning.

Immunohistochemical staining. Paraffin sections (5- $\mu \mathrm{m}$-thick) from tissues were dewaxed in xylene, rehydrated in graded alcohol and endogenous peroxidase activity was blocked by infiltrating in $0.3 \%$ hydrogen peroxide. Then, to enhance the accessibility of the antigen, the sections were treated with $0.1 \mathrm{M}$ citrate buffer ( $\mathrm{pH}$ 6.0) and heated to $121^{\circ} \mathrm{C}$ in an autoclave for $3 \mathrm{~min}$. After cooling, hydrogen peroxide (0.3\%) was used to block endogenous peroxide activity for $20 \mathrm{~min}$. Next the sections were rinsed in phosphate-buffered saline (PBS) (pH 7.2) and then incubated with the DTX3L (cat. no. sc-100627; Santa Cruz Biotechnology, Inc., Santa Cruz, CA, USA) and Ki-67 (cat. no. sc-23900; Santa Cruz Biotechnology, Inc.) antibodies for $2 \mathrm{~h}$ at room temperature. All slides were processed using immunoglobulin $\mathrm{IgG}$ as a secondary antibody. After being washed in PBS, the peroxidase reaction was visualized by incubating the sections with DAB $(0.02 \%$ diaminobenzidine tetrahydrochloride, $0.1 \%$ phosphate buffer solution and $3 \% \mathrm{H}_{2} \mathrm{O}_{2}$ ). Finally, the sections were counterstained with hematoxylin, dehydrated with graded alcohol and mounted in resin mount. A microscope was used to observe the stained sections. The immunostaining was evaluated separately by two independent pathologists. For the assessment of DTX3L and $\mathrm{Ki}-67$, five high-power fields were randomly chosen and at least 300 cells were counted in each section. The percentage of tumor cells that stained positive was scored as follows: 1 , $0-49 \%$ positive tumor cells; $2,50-74 \%$ positive tumor cells; and $3,75-100 \%$ positive tumor cells. The intensity of staining was estimated and scored as follows: 0 , no staining; 1 , weak staining; 2 , moderate staining; and 3, strong staining. Then, we combined scores from the two scales and classified each section as follows: $0-4.5$ was classified as low expression and 4.5-9 was classified as high expression.

Western blot analysis. Proteins isolated from tissues and cell samples were promptly homogenized in lysis buffer which contained $1 \mathrm{M}$ Tris- $\mathrm{HCl} \mathrm{pH} 7.5,1 \%$ Triton X-100, $1 \%$ Nonidet P-40 (NP-40), $10 \%$ sodium dodecyl sulfate (SDS), $0.5 \%$ sodium deoxycholate, $0.5 \mathrm{M}$ EDTA, $10 \mu \mathrm{g} / \mathrm{ml}$ leupeptin, $10 \mu \mathrm{g} / \mathrm{ml}$ aprotinin, and $1 \mathrm{mM}$ PMSF and then centrifuged for $30 \mathrm{~min}$ at $10,000 \mathrm{xg}$ to collect the supernatant liquid. The supernatants were stored at $-80^{\circ} \mathrm{C}$ until use. The protein concentrations were measured with a Bio-Rad protein assay (Bio-Rad, Hercules, CA, USA). Equal amount of protein from each sample was separated by SDS-polyacrylamide gel electrophoresis (PAGE) and then transferred onto a PVDF membrane (Millipore, Bedford, MA, USA). After being blocked in $5 \%$ non-fat milk in TBST [150 mM NaCl, $20 \mathrm{mM}$ Tris (pH 7.4) and $0.05 \%$ Tween-20] for $2 \mathrm{~h}$, the membranes were first incubated with primary antibodies overnight at $4^{\circ} \mathrm{C}$. The primary antibodies used in this report included the followed: anti-glyceraldehyde 3-phosphate dehydrogenase (GAPDH) (cat. no. sc-47724; 1:3,000), anti-DTX3L (cat. no. sc-100627; 1:500), E-cadherin (cat. no. sc-8426; 1:500), vimentin (cat. no. sc-6260; 1:1,000) (all from Santa Cruz Biotechnology, Inc.), anti-caspase-3 (cleaved) (cat. no. 9661; 1:1,000), and anti-PARP (cleaved) (cat. no. 5625; 1:1,000) (both from Cell Signaling Technology, Danvers, MA, USA). After washing for three times, $5 \mathrm{~min}$ each, the membranes were then incubated with horseradish peroxidase-conjugated human anti-mouse (cat. no. A11126) or anti-rabbit (cat. no. 71-2700) antibodies (1:1,000; Pierce, Rockford, IL, USA) as the secondary antibody at room temperature for $2 \mathrm{~h}$. Finally, the membranes were detected with ECL detection systems. The experiments were implemented in three independent reactions.

Cell cultures and transient transfection. Normal human gliocyte HEB cells and human glioblastoma cell lines including U251MG, U87MG and A172 were obtained from the Cell Library of the Chinese Academy of Sciences. Cells were cultured in Dulbecco's modified Eagle's medium (DMEM) (Gibco-BRL, Grand Island, NY, USA) with $10 \%$ fetal bovine serum (FBS), $2 \mathrm{mM}$ L-glutamine, and $100 \mathrm{U} / \mathrm{ml}$ penicillinstreptomycin mixture (Gibco-BRL) at $37^{\circ} \mathrm{C}$ in a humidified incubator containing $5 \% \mathrm{CO}_{2}$. The medium was changed every 2-3 days, and cultures were split using $0.25 \%$ trypsin. Lipofectamine 2000 (Invitrogen, Carlsbad, CA, USA) was used to transfect the U251MG cells with siRNAs according to the manufacturer's instructions. Four siRNAs targeting the DTX3L gene were designed and synthesized, and western blot analysis was used to identify the most effective siRNA for the further experiments. The sequences are as follows: 5'-CGTATTAGG AGTCTCAGAT-3', 5'-GATGGACATTGATAGCGAT-3', 5'-TGATTTAATGCCAGTCTAA-3' and 5'-CAATTACAT GATGAATGTA-3'. Cells were transfected with DTX3L-siRNA or control-siRNA and then collected after $24-48 \mathrm{~h}$ for the following assays.

Multicellular tumor spheroid formation assays. U251MG cells transfected with DTX3L siRNA and control siRNA were seeded at a density of $4 \times 10^{5}$ cells/well in 6-well plates. Then, the cells were washed with PBS and stained with $0.5 \%(\mathrm{w} / \mathrm{v})$ crystal violet in 70\% ethanol after being cultured for 4-6 days. Finally, the amount of multicellular tumor spheroids from the indicated fields $(>10)$ was measured under a light microscope.

Cell growth assay. To evaluate the effect of transfection of DTX3L (control or siRNA), the cells were plated in 96-well plates at a density of $2 \times 10^{4} /$ well in a volume of $100 \mu 1$ in medium with $10 \%$ FBS. Each well was transfected for the indicated times after 24 h. Cell Counting Kit-8 (Dojindo, Kumamoto, Japan) reagents were added to each well for 2-h incubation at $37^{\circ} \mathrm{C}$, after which absorbance at the wavelength of $450 \mathrm{~nm}$ was measured in an automated plate reader. 
A

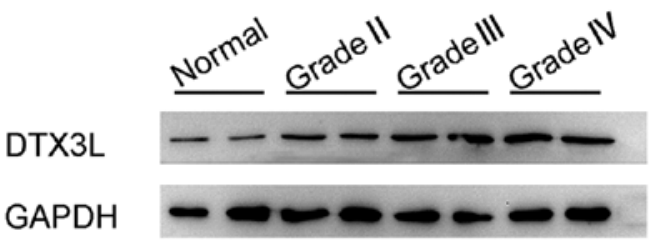

B

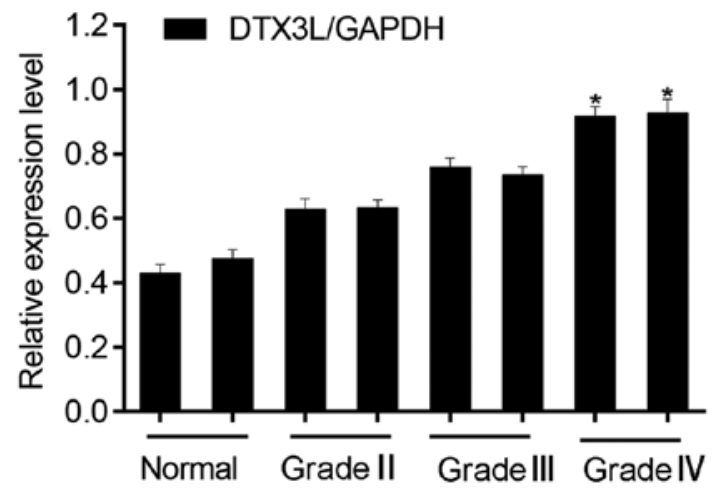

C

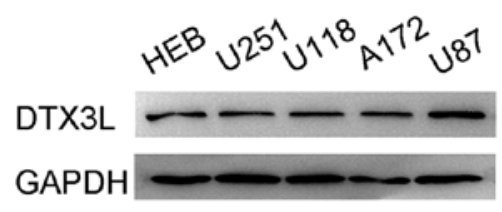

D

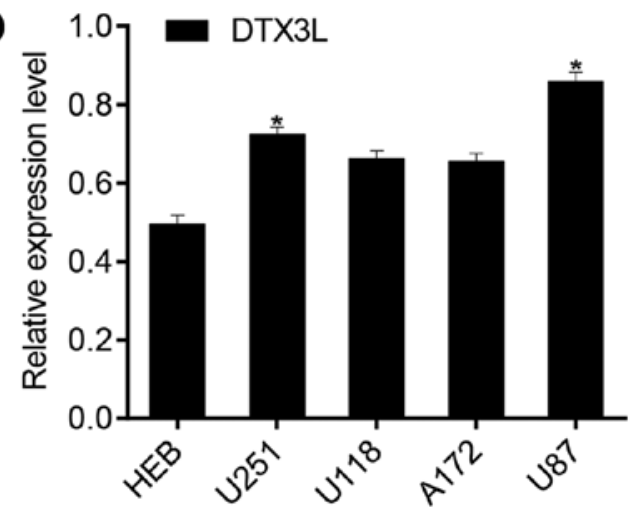

Figure 1. Expression of Deltex-3-like (DTX3L) in human glioma tissues and cells. (A and C) Western blot analysis of DTX3L expression in glioma tissues (grades II-IV), normal brain tissues, HEB, and U251MG, A172, U87MG and U118 glioma cells. Glyceraldehyde 3-phosphate dehydrogenase (GAPDH) was used as a control for protein load and integrity. (B and D) The bar charts show the ratio of DTX3L protein to GAPDH for the above by densitometry. Data are expressed as the mean $\pm \mathrm{SD}$ of three independent experiments. ${ }^{*} \mathrm{P}<0.05$.

Wound-healing assays. U87MG cells were seeded at a density of $2 \times 10^{6}$ in 6-well plates and incubated in DMEM supplemented with $10 \%$ FBS for $24 \mathrm{~h}$. The cells were then transfected with DTX3L siRNA and control siRNA for $36 \mathrm{~h}$ and subsequently serum starved for $12 \mathrm{~h}$. A straight scratch was made in each well with a $10-\mu 1$ micropipette tip at the same time. After being washed three times with PBS, the cells were cultured in 5\% FBS-DMEM in a humidified atmosphere of $5 \% \mathrm{CO}_{2}$ at $37^{\circ} \mathrm{C}$, and images of the width of the wound were captured under a microscope at 0-, 24- and 48-h time-points.

Transwell migration assay. U87MG cells were transfected with DTX3L siRNA and control siRNA for $48 \mathrm{~h}$ and then resuspended in DMEM supplemented with $0.1 \%$ bovine serum albumin. Cells at a density of $1 \times 10^{5}$ were added to the upper chambers of 24-well Transwell plates ( $8-\mu$ m pore size; Corning, Inc., Corning, NY, USA), and complete medium was added to the lower chambers. After being incubated for $36 \mathrm{~h}$ at $37^{\circ} \mathrm{C}$, the cells remaining in the upper chamber (non-migrated) were removed, and the ones on the bottom chamber (migrated) were fixed and stained with crystal violet to visualize the nuclei. The number of cells that had migrated through the polycarbonate membrane was counted under x200 magnification. All experiments were performed in triplicate and repeated twice.

Annexin $V$ analysis. U87MG cells were transfected with the indicated siRNA or plasmid and subsequently treated with TMZ for $4 \mathrm{~h}$ as indicated. Then after being separated with trypsin, the cells were washed three times and stained with FITC-Annexin V (Biosource, Camarillo, CA, USA) for $15 \mathrm{~min}$ and then finally detected on a FacsCalibur (BD Biosciences, San Jose, CA, USA).

Statistical analysis. All of the numerical data were analyzed using the SPSS 17.0 software package (SPSS, Inc., Chicago,
IL, USA). The statistical significance of the correlations between DTX3L and clinicopathologic features were analyzed by the Chi-square $\left(\chi^{2}\right)$ test. Univariate survival analysis was performed using the Kaplan-Meier method and curves were compared using the log-rank test. Multivariate survival analysis was carried out using Cox's proportional hazards regression model and the risk ratio and its $95 \%$ confidence interval were recorded for every marker. P-value $<0.05$ was considered to indicate a statistically significant difference. All values are presented as mean \pm SEM and each experiment was carried out three times.

\section{Results}

Identification of DTX $3 L$ expression in glioma. To reveal the potential role of DTX3L in glioma progression, we firstly used western blotting to analyze the expression of DTX3L in a panel of glioma specimens, including two normal brain tissue samples and six glioma samples. We noted that DTX3L protein expression was significantly increased in high-grade glioma tissues compared with that observed in the low-grade glioma tissues and normal brain tissues (Fig. 1A and B). Then, we also examined the expression of DTX3L in normal human gliocyte HEB cells and glioma cell lines, U87MG, U251MG, A172 and U118. As expected, DTX3L was highly expressed in the glioma cell lines (Fig. 1C and D) compared to that noted in the HEB cells. Next, immunohistochemistry was used to analyze DTX3L expression and $\mathrm{Ki}-67$ expression in 96 glioma samples to confirm the association of DTX3L with glioma progression. DTX3L expression was increased as the degree of malignancy was increased (Fig. 2A). In addition, Spearman's correlation coefficient demonstrated a positive correlation between DTX3L and Ki-67 expression ( $r=0.568$, $\mathrm{P}<0.01)$ (Fig. 2B). 
A
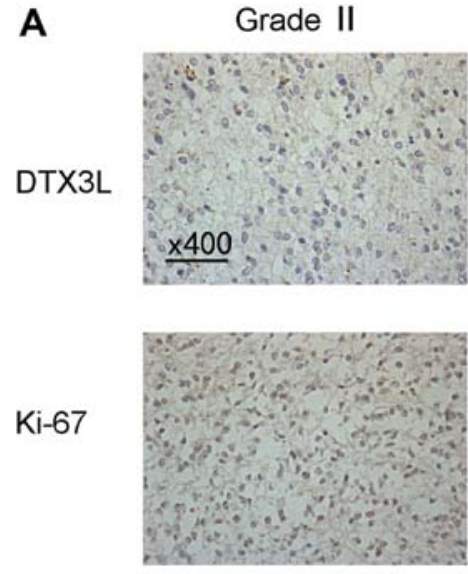

Grade III
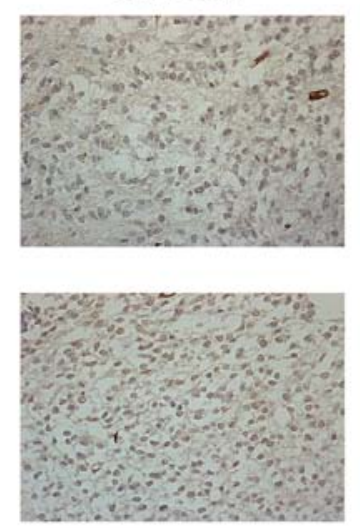

Grade IV
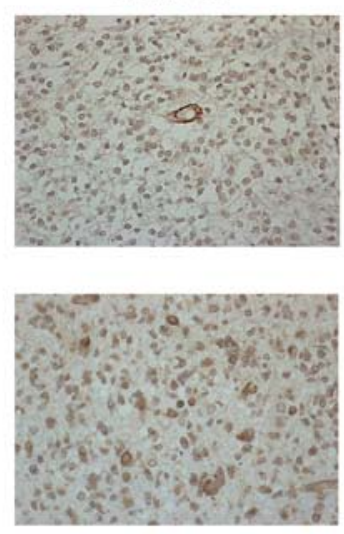

B

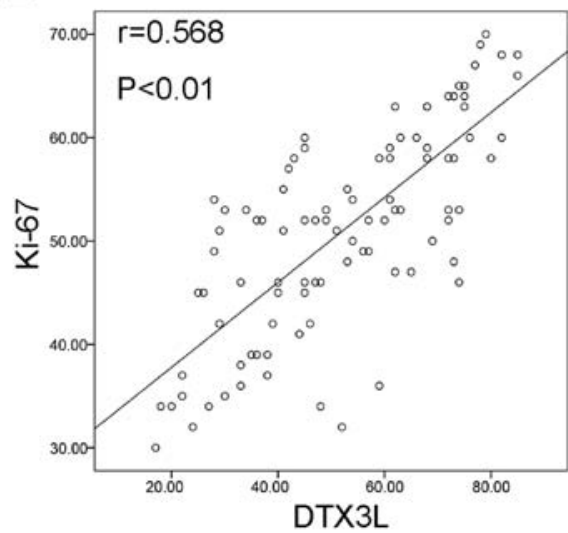

C

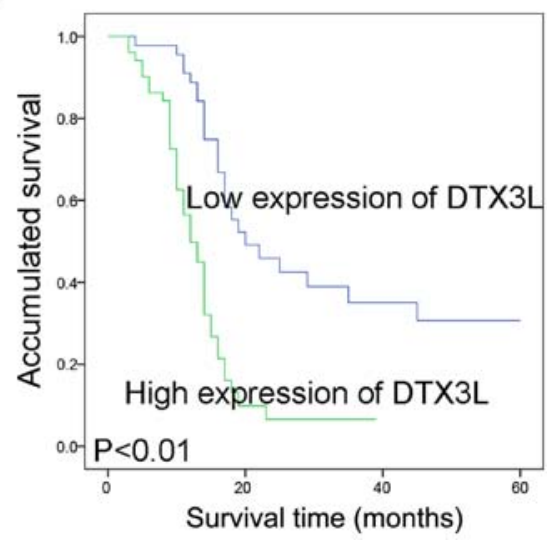

Figure 2. The correlation between Deltex-3-like (DTX3L) and the grade of glioma, Ki-67 and glioma outcome. (A) Paraffin-embedded glioma tissue sections (including grades II-IV) were stained with anti-DTX3L antibodies and anti-Ki-67 antibodies, and counterstained with hematoxylin (SP x400). (B) The relationship between DTX3L and Ki-67. (C) Kaplan-Meier survival curve for patterns of patients with glioma and the expression of DTX3L in 96 patients with glioma. Patients in the high-expression DTX3L group had an apparently shorter overall survival $(\mathrm{P}<0.01)$.

DTX3L expression and patient survival. To further explore the correlation between clinicopathologic and pathophysiological features of glioma and DTX3L expression, the immunohistochemical results of the 96 glioma specimens are listed in Table I. We found that DTX3L expression was significantly correlated with clinicopathologic grade $(\mathrm{P}=0.002)$ and $\mathrm{Ki}-67$ expression ( $\mathrm{P}=0.027)$. However, no significant correlation was observed between DTX3L expression and patient age, sex, tumor location, type of surgery, or tumor size in the 96 glioma cases. Multivariate Cox regression analysis indicated that DTX3L expression is an independent predictor of survival $(\mathrm{P}=0.022)$ (Table II). Furthermore, Kaplan-Meier survival curves indicated that upregulation of DTX3L was significantly correlated with a shortened overall survival $(\mathrm{P}<0.01)$ (Fig. 2C).

Knockdown of DTX3L by RNA interference. We selected U87MG cells for the siRNA experiment, as this cell line expresses a high level of endogenous DTX3L. To further study the potential function of DTX3L in glioma cell proliferation, we used four siRNAs against DTX3L expression to test their interference efficiency and the result showed that DTX3L protein levels were significantly decreased in the cells transfected with siDTX3L-1 compared with the level noted in the cells transfected with the control siRNA (Fig. 3A and B). It has been reported that U87MG cells have the ability to spontaneously form multicellular tumor spherioids $(14,15)$; thus we speculated that DTX3L has an effect on tumor spheroid formation. We transfected U87MG cells with DTX3L-siRNA or control-siRNA. Knockdown of DTX3L resulted in an obvious decrease in multicellular tumor spheroids (Fig. 3C). In addition, we examined the effect of DTX3L silencing on the cell growth rate. As expected, knockdown of DTX3L was able to inhibit the cell growth rate (Fig. 3D). Taken together, we demonstrated that DTX3L silencing coutributed to a reduction in the proliferation of the glioma cells.

Decreased cell invasion in the DTX3L-depleted glioma cells. Since a previous study reported that DTX3L significantly contributes to the migration and invasion of melanoma cells (12), we also investigated whether silencing of DTX3L could inhibit glioma cell migration and invasion. We analyzed the potential ability of DTX3L to induce cell motility using wound-healing and Transwell assays. In the wound-healing assay, the cell migration was reduced in the DTX3L-siRNA group compared with that in the control group, which indicated that the migratory ability of the U87MG cells transfected with DTX3L-siRNA was much weaker than that in the cells transfected with the control-siRNA (Fig. 4A and B). In addition, the results of the Transwell migration assay also showed that the number of U87MG cells which passed through the 
A
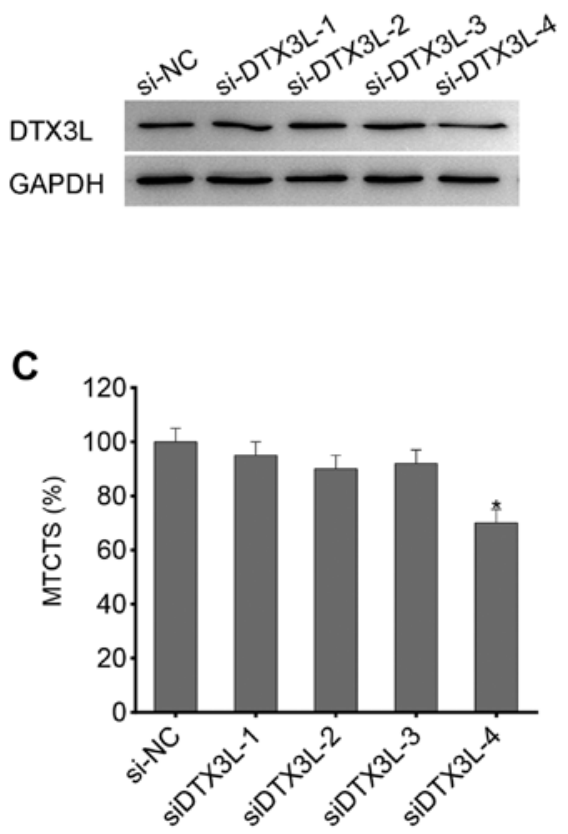

B

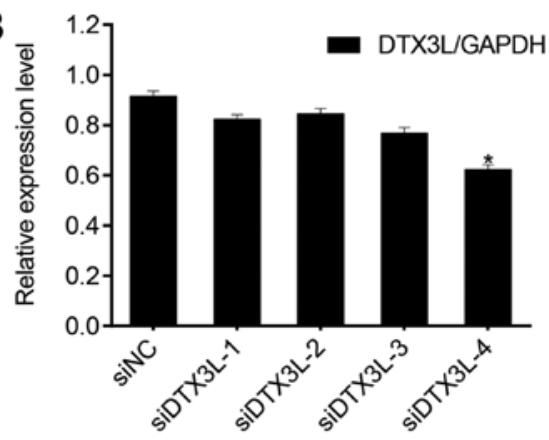

D $\quad 2.5] \rightarrow$ siDTX $3 \mathrm{~L}-4$

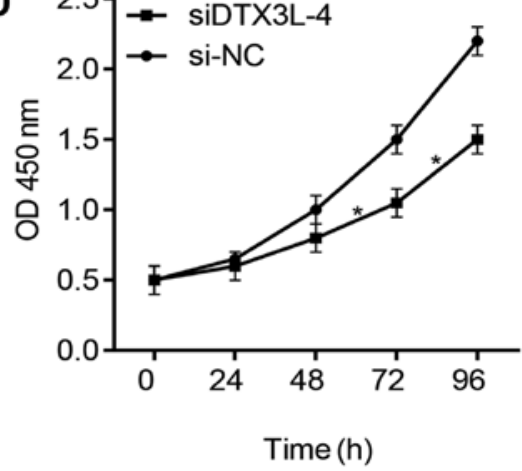

Figure 3. Effect of Deltex-3-like (DTX3L) downregulation on glioma cell growth. (A) Western blot analysis was used to analyze DTX3L expression after transfection with DTX3L-siRNA in U87MG cells and the results showed that siDTX3L-4 achieved the most effective knockout effect. (B) The bar chart shows the ratio of DTX3L protein to glyceraldehyde 3-phosphate dehydrogenase (GAPDH) for the above by densitometry. Data are expressed as the mean \pm SD. "P $<0.05$ compared with the control. (C) The histogram shows the mean \pm SD values of MCTS formation from three independent experiments and each experiment was conducted in duplicate. Data are expressed as the percentage of MCTS relative to the control cells. Student's $t$-test, ${ }^{\text {"P }}<0.05$. (D) The graph exhibits the growth curves of U87MG cells transfected with DTX3L (control or siRNA). "P<0.05.

A
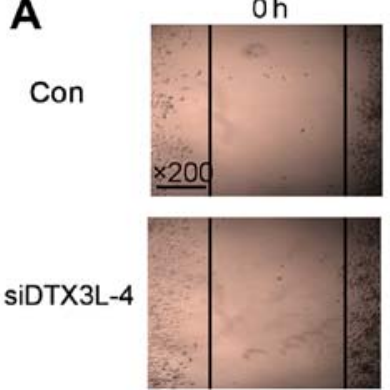

C

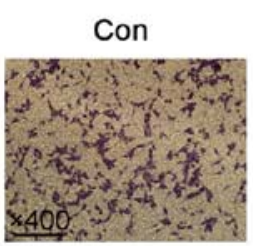

$24 \mathrm{~h}$
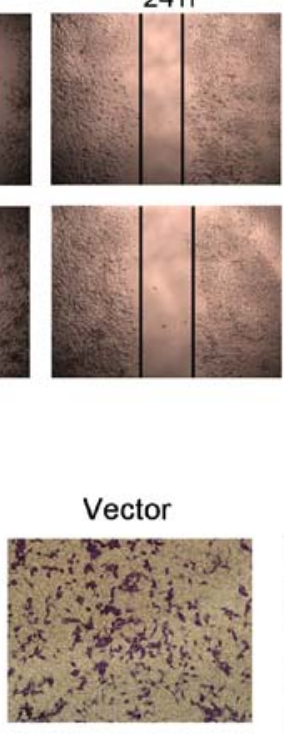

E

E-cadherin

Vimentin

GAPDH
$48 \mathrm{~h}$

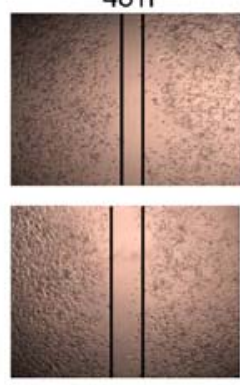

siDTX3L-4

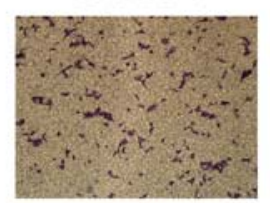

(1) 4
B

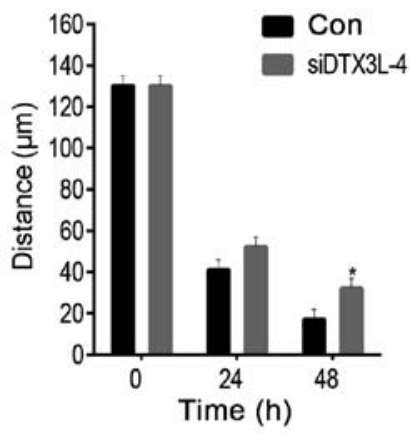

D

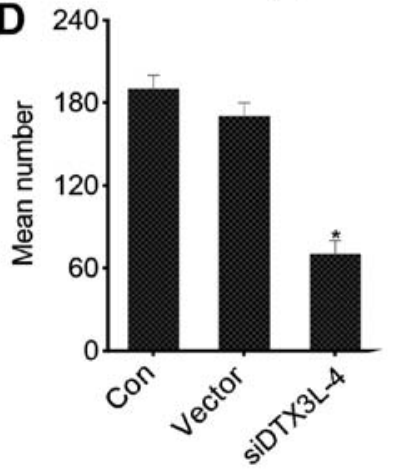

Figure 4. Silencing of Deltex-3-like (DTX3L) inhibits the migration of glioma cells. (A and B) U87MG cells transfected with DTX3L-siRNA and controlsiRNA were examined in wound-healing assays. Images were captured at 0 , and $48 \mathrm{~h}$ using an inverted Leica phase-contrast microscope (x200 magnification). ${ }^{*} \mathrm{P}<0.05$. (C and D) Transwell assays indicate the effect of DTX3L expression on glioma cell migration ability. Each time-point was determined from three independent experiments. ${ }^{*} \mathrm{P}<0.05$. (E) Western blot analysis of E-cadherin and vimentin in the control, vector and siDTX3L cells. 
Table I. Correlation between DTX3L expression and the clinicopathological characteristics of the 96 glioma specimens.

\begin{tabular}{|c|c|c|c|c|c|}
\hline \multirow[b]{2}{*}{ Variables } & \multirow[b]{2}{*}{ Total } & \multicolumn{2}{|c|}{ DTX3L expression } & \multirow[b]{2}{*}{$\chi^{2}$ value } & \multirow[b]{2}{*}{ P-value } \\
\hline & & $\begin{array}{c}\text { Low } \\
(\text { score }<5)(\end{array}$ & $\begin{array}{c}\text { High } \\
(\text { score } \geq 5)\end{array}$ & & \\
\hline \multicolumn{6}{|l|}{ Age (years) } \\
\hline$<50$ & 48 & 25 & 23 & 1.046 & 0.306 \\
\hline$\geq 50$ & 48 & 20 & 28 & & \\
\hline \multicolumn{6}{|l|}{ Sex } \\
\hline Female & 39 & 19 & 20 & 0.090 & 0.765 \\
\hline Male & 57 & 26 & 31 & & \\
\hline \multicolumn{6}{|l|}{ Tumor location } \\
\hline Frontal & 30 & 18 & 12 & 3.895 & 0.420 \\
\hline Parietal & 12 & 6 & 6 & & \\
\hline Occipital & 13 & 6 & 7 & & \\
\hline Temporal & 28 & 10 & 18 & & \\
\hline Unknown & 13 & 5 & 8 & & \\
\hline \multicolumn{6}{|l|}{ Surgery } \\
\hline Biopsy & 17 & 8 & 9 & 0.014 & 0.993 \\
\hline Total resection & 55 & 26 & 29 & & \\
\hline Subtotal section & 24 & 11 & 13 & & \\
\hline \multicolumn{6}{|l|}{ Tumor size $(\mathrm{cm})$} \\
\hline$<4$ & 54 & 28 & 26 & 1.228 & 0.268 \\
\hline$\geq 4$ & 42 & 17 & 25 & & \\
\hline \multicolumn{6}{|l|}{ WHO grade } \\
\hline II & 28 & 19 & 9 & 12.268 & $0.002^{\mathrm{a}}$ \\
\hline III & 43 & 21 & 22 & & \\
\hline IV & 25 & 5 & 20 & & \\
\hline \multicolumn{6}{|l|}{ Ki-67 expression } \\
\hline Low & 44 & 26 & 18 & 4.868 & $0.027^{\mathrm{a}}$ \\
\hline High & 52 & 19 & 33 & & \\
\hline
\end{tabular}

Statistical analyses were performed by the Pearson's $\chi^{2}$-test. ${ }^{\text {a }}<0.05$ was considered significant.

membrane onto the lower chamber was markedly less in the the DTX3L-siRNA-transfected cells compared to that noted in the control-siRNA-transfected cells (Fig. 4C and D). Next, in order to validate these results, we assessed the protein levels of E-cadherin and vimentin, which are markers of epithelialmesenchymal transition (EMT) $(16,17)$ and contribute to tumor metastasis, in the DTX3L-siRNA group and the negative control group by western blot analysis. As shown in Fig. 4C, in the DTX3L-siRNA group, E-cadherin expression was upregulated, while vimentin expression exhibited an opposite trend. These observations confirm that DTX3L was associated with glioma migration and invasion.

Loss of DTX3L expression sensitizes glioma cells to a chemotherapeutic drug. TMZ is the main therapeutic strategy for patients suffering from gliomas $(18,19)$. In this study, we analyzed whether loss of DTX3L expression could enhance
Table II. Contribution of various potential prognostic factors to survival by Cox regression analysis of 96 glioma specimens.

\begin{tabular}{lccc}
\hline Characteristics & Hazard ratio & $95 \%$ CI & P-value \\
\hline Age (years) & 1.271 & $0.753-2.146$ & 0.370 \\
Sex & 1.597 & $0.886-2.878$ & 0.119 \\
Tumor location & 0.985 & $0.820-1.184$ & 0.874 \\
Tumor size & 1.054 & $0.607-1.830$ & 0.852 \\
Surgery & 1.074 & $0.712-1.619$ & 0.734 \\
WHO grade & 1.832 & $1.269-2.647$ & $0.001^{\mathrm{a}}$ \\
DTX3L expression & 2.038 & $1.110-3.744$ & $0.022^{\mathrm{a}}$ \\
Ki-67 expression & 2.150 & $1.149-4.022$ & $0.017^{\mathrm{a}}$ \\
\hline
\end{tabular}

Statistical analyses were performed by the Cox regression analysis ${ }^{a} \mathrm{P}<0.05$ was considered significant. $\mathrm{CI}$, confidence interval.

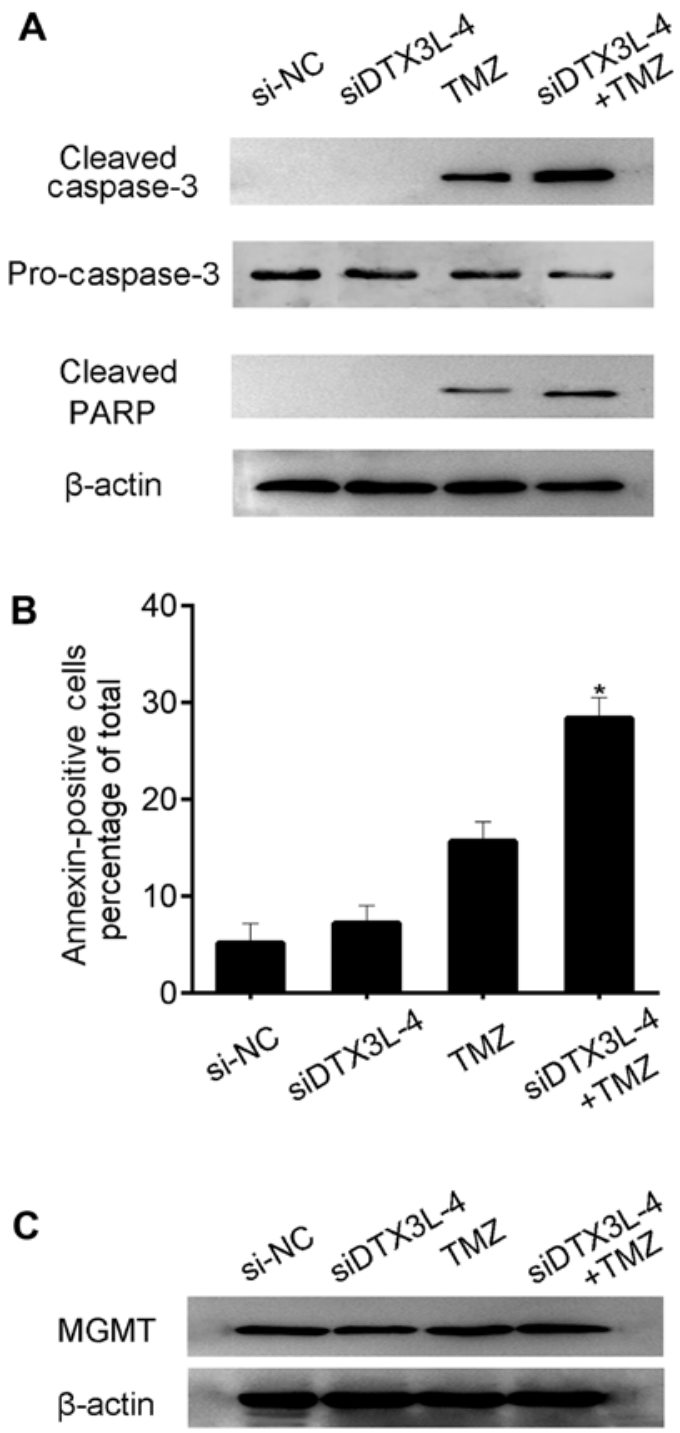

Figure 5. Knockdown of Deltex-3-like (DTX3L) sensitizes glioma cells to temozolomide (TMZ). (A) U87MG cells transfected with DTX3L-siRNA and control-siRNA were treated with TMZ as indicated for $4 \mathrm{~h}$. Whole cell lysates were collected, and western blot was used to analyze the levels of cleaved caspase-3, cleaved PARP and glyceraldehyde 3-phosphate dehydrogenase (GAPDH). (B) The percentages of Annexin V-positive U87MG cells treated as indicated were determined. ${ }^{*} \mathrm{P}<0.05$. (C) Western blotting was used to assess the protein level of MGMT in the cells treated as indicated. 
the cytotoxic effects of TMZ in U87MG cells. We first transfected U87MG cells with DTX3L-siRNA or control-siRNA and subsequently treated the U87MG cells with TMZ. Next western blot analysis was performed to detect the cleavage of caspase-3 and PARP in the U87MG cells. As expected, knockdown of DTX3L contributed to increased expression of caspase-3 and PARP (Fig. 5A). Meanwhile, the percentages of Annexin V-positive U87MG cells in the different treatment groups were determined. The percentage of apoptotic cells was increased in the DTX3L-siRNA + TMZ group compared with that in the DTX3L-siRNA and TMZ treatment alone groups. Our data revealed that knockdown of DTX3L expression by siRNA facilitated glioma cell apoptosis induced by TMZ (Fig. 5B). Since enzyme $\mathrm{O}^{6}$-methylguanine-DNA methyltransferase (MGMT) has been reported to suppress TMZ sensitivity, we further examined the protein level of MGMT. The results showed that MGMT was not apparently alter in the experiment (Fig. 5C).

\section{Discussion}

Malignant gliomas are the most prevalent brain tumors with a poor survival despite maximal therapy $(20,21)$. Although traditional treatments for glioma such as surgical resection, chemotherapy and radiotherapy have a beneficial effect on patient survival, the prognosis of glioma patients remains poor $(4,21,22)$. This poor prognosis is mainly due to loss of control of the primary tumor and widespread invasion of tumor cells throughout the normal brain $(21,23)$. Therefore, elucidation of the molecular biological mechanisms underlying glioma pathogenesis are urgently needed.

DTX3L is a member of the Deltex (DTX) family (DTX1, DTX2 and DTX3) (7). Much research suggests that DTX3L is involved in the occurrence and development of a variety of solid cancers $(12,13)$. DTX3L, as a component of the BAL/ DTX3L complex, has been reported to be involved in host response diffuse large B-cell lymphomas by regulating the nucleocytoplasmic trafficking of transcription factors (24). An additional study found that DTX3L participates in the DNA damage response pathway following exposure to genotoxic agents via the post-translational modification of histone H4 (10). A proteomic study showed that DTX3L has an effect on tumorigenesis via influencing the nuclear activities of STAT1 by antagonistically regulating the tyrosine phosphorylation of STAT1 on Y701 in cooperation with ARTD9 (13). Moreover, elevated DTX3L was found to contribute to cellular metastasis during melanoma pathogenesis (12). On the basis of these findings, we inferred that DTX3L may be involved in the progression of tumors. Thus, in the present study, we explored the potential function of DTX3L in glioma progression.

Recent studies have reported that aberrant functioning of DTX3L is considered to cause cell migration and dysregulated cell growth $(12,14)$. In this study, we showed that depletion of DTX3L by RNA interference suppressed glioma cell migration and the DTX3L protein level was negatively correlated with E-cadherin expression, which is a marker of epithelial-mesenchymal transition (EMT) and contributes to cancer metastasis (25). As known, during carcinogenesis, tumor cell resistance to apoptosis contributes to the development and progression of cancers (26). TMZ is considered as a first-line chemotherapeutic drug for the treatment of glioma patients (27). Accordingly, we aimed to ascertain whether DTX3L has an effect on sensitizing glioma cells to TMZ-induced apoptosis. As expected, our data revealed that knockdown of DTX3L expression by siRNA facilitated glioma cell apoptosis induced by TMZ. In conclusion, this is the first study to demonstrate that the DTX3L level is significantly increased in human glioma tissues and DTX3L upregulation is correlated with glioma grade and poor prognosis of glioma. Furthermore, the silencing of DTX3L by RNA interference inhibited the growth and migration of glioma cells as well as induced cell apoptosis. Based on these findings, we propose that DTX3L may serve as a potential prognostic biomarker and a therapeutic target for glioma. However, the detailed mechanism underlying the association between DTX3L and the development of glioma needs further investigation.

\section{Acknowledgements}

This study was supported by the National Natural Science Foundation of China Grants (nos. 81272789 and 81572491).

\section{References}

1. Roy LO, Poirier MB and Fortin D: Transforming growth factor-beta and its implication in the malignancy of gliomas. Target Oncol 10: 1-14, 2015.

2. Osswald M, Jung E, Sahm F, Solecki G, Venkataramani V, Blaes J, Weil S, Horstmann H, Wiestler B, Syed M, et al: Brain tumour cells interconnect to a functional and resistant network. Nature 528: 93-98, 2015.

3. Tao T, Cheng C, Ji Y, Xu G, Zhang J, Zhang L and Shen A: Numbl inhibits glioma cell migration and invasion by suppressing TRAF5-mediated NF- $\mathrm{B}$ B activation. Mol Biol Cell 23: 2635-2644, 2012

4. Bai RY, Staedtke V and Riggins GJ: Molecular targeting of glioblastoma: Drug discovery and therapies. Trends Mol Med 17: 301-312, 2011.

5. Qazi MA, Vora P, Venugopal C, McFarlane N, Subapanditha MK, Murty NK, Hassell JA, Hallett RM and Singh SK: A novel stem cell culture model of recurrent glioblastoma. J Neurooncol 126: 57-67, 2016

6. Ding Z, Liu Y, Yao L, Wang D, Zhang J, Cui G, Yang X, Huang X, Liu F and Shen A: Spyl induces de-ubiquitinating of RIP1 arrest and confers glioblastoma's resistance to tumor necrosis factor (TNF- $\alpha$ )-induced apoptosis through suppressing the association of CLIPR-59 and CYLD. Cell Cycle 14: 2149-2159, 2015.

7. Takeyama K, Aguiar RC, Gu L, He C, Freeman GJ, Kutok JL, Aster JC and Shipp MA: The BAL-binding protein BBAP and related Deltex family members exhibit ubiquitin-protein isopeptide ligase activity. J Biol Chem 278: 21930-21937, 2003.

8. Zhang Y, Mao D, Roswit WT, Jin X, Patel AC, Patel DA, Agapov E, Wang Z, Tidwell RM, Atkinson JJ, et al: PARP9-DTX3L ubiquitin ligase targets host histone $\mathrm{H} 2 \mathrm{BJ}$ and viral $3 \mathrm{C}$ protease to enhance interferon signaling and control viral infection. Nat Immunol 16: 1215-1227, 2015.

9. Holleman J and Marchese A: The ubiquitin ligase deltex-31 regulates endosomal sorting of the $\mathrm{G}$ protein-coupled receptor CXCR4. Mol Biol Cell 25: 1892-1904, 2014.

10. Yan Q, Dutt S, Xu R, Graves K, Juszczynski P, Manis JP and Shipp MA: BBAP monoubiquitylates histone H4 at lysine 91 and selectively modulates the DNA damage response. Mol Cell 36: 110-120, 2009.

11. Yan Q, Xu R, Zhu L, Cheng X, Wang Z, Manis J and Shipp MA: BAL1 and its partner E3 ligase, BBAP, link Poly(ADP-ribose) activation, ubiquitylation, and double-strand DNA repair independent of ATM, MDC1, and RNF8. Mol Cell Biol 33: 845-857, 2013.

12. Thang ND, Yajima I, Kumasaka MY, Iida M, Suzuki T and Kato M: Deltex-3-like (DTX3L) stimulates metastasis of melanoma through FAK/PI3K/AKT but not MEK/ERK pathway. Oncotarget 6: 14290-14299, 2015. 
13. Bachmann SB, Frommel SC, Camicia R, Winkler HC, Santoro R and Hassa PO: DTX3L and ARTD9 inhibit IRF1 expression and mediate in cooperation with ARTD8 survival and proliferation of metastatic prostate cancer cells. Mol Cancer 13: 125, 2014

14. Kolchinsky A and Roninson IB: Drug resistance conferred by MDR1 expression in spheroids formed by glioblastoma cell lines. Anticancer Res 17: 3321-3327, 1997.

15. Günther W, Pawlak E, Damasceno R, Arnold H and Terzis AJ: Temozolomide induces apoptosis and senescence in glioma cells cultured as multicellular spheroids. Br J Cancer 88: 463-469, 2003.

16. Stoops SL, Waterson AG, An H, Deane N, Daniels JS, Morrison R, Engers JL, Beauchamp D and Lindsley CW: Discovery and characterization of a small molecule that restores E-cadherin expression in cancer cell lines via a new mechanism. Probe Reports from the NIH Molecular Libraries Program (Internet). Bethesda (MD): National Center for Biotechnology Information (US); 2010-2012 Dec 13 (updated 2013 Mar 22). Available from: https://www.ncbi.nlm.nih.gov/books/NBK344134/

17. Lindsay CR, Le Moulec S, Billiot F, Loriot Y, Ngo-Camus M, Vielh P, Fizazi K, Massard C and Farace F: Vimentin and $\mathrm{Ki}-67$ expression in circulating tumour cells derived from castrate-resistant prostate cancer. BMC Cancer 16: 168, 2016.

18. Castro GN, Cayado-Gutiérrez N, Zoppino FC, Fanelli MA, Cuello-Carrión FD, Sottile M, Nadin SB and Ciocca DR: Effects of temozolomide (TMZ) on the expression and interaction of heat shock proteins (HSPs) and DNA repair proteins in human malignant glioma cells. Cell Stress Chaperones 20: 253-265, 2015.

19. Zhuang D, Liu Y, Mao Y, Gao L, Zhang H, Luan S, Huang F and Li Q: TMZ-induced PrPc/par-4 interaction promotes the survival of human glioma cells. Int J Cancer 130: 309-318, 2012.
20. Ogbomo H, Cinatl J Jr, Mody CH and Forsyth PA: Immunotherapy in gliomas: Limitations and potential of natural killer (NK) cell therapy. Trends Mol Med 17: 433-441, 2011.

21. Rich JN and Bigner DD: Development of novel targeted therapies in the treatment of malignant glioma. Nat Rev Drug Discov 3: 430-446, 2004.

22. Ding Z, Liu X, Liu Y, Zhang J, Huang X, Yang X, Yao L, Cui G and Wang D: Expression of far upstream element (FUSE) binding protein 1 in human glioma is correlated with c-Myc and cell proliferation. Mol Carcinog 54: 405-415, 2015.

23. Chen J, Sun J, Yang L, Yan Y, Shi W, Shi J, Huang Q, Chen J and Lan Q: Upregulation of B23 promotes tumor cell proliferation and predicts poor prognosis in glioma. Biochem Biophys Res Commun 466: 124-130, 2015.

24. Juszczynski P, Kutok JL, Li C, Mitra J, Aguiar RC and Shipp MA: BAL1 and BBAP are regulated by a gamma interferon-responsive bidirectional promoter and are overexpressed in diffuse large B-cell lymphomas with a prominent inflammatory infiltrate. Mol Cell Biol 26: 5348-5359, 2006.

25. Xu H, Tian Y, Yuan X, Liu Y, Wu H, Liu Q, Wu GS and Wu K: Enrichment of CD44 in basal-type breast cancer correlates with EMT, cancer stem cell gene profile, and prognosis. Onco Targets Ther 9: 431-444, 2016

26. Li RY, Chen LC, Zhang HY, Du WZ, Feng Y, Wang HB, Wen JQ, Liu X, Li XF, Sun Y, et al: MiR-139 inhibits Mcl-1 expression and potentiates TMZ-induced apoptosis in glioma. CNS Neurosci Ther 19: 477-483, 2013.

27. Yan Y, Xu Z, Dai S, Qian L, Sun L and Gong Z: Targeting autophagy to sensitive glioma to temozolomide treatment. J Exp Clin Cancer Res 35: 23, 2016 